\title{
SIMULAÇÃO EM USO DOS PISOS DE MADEIRA DE Eucalyptus sp E Corymbia maculata
}

\author{
Marcelo Martins ${ }^{1}$, José Reinaldo Moreira da Silva², José Tarcísio Lima ${ }^{3}$, \\ Marcos Tadeu Tiburcio Gonçalves ${ }^{4}$, Alexandre Petusk Filipe ${ }^{5}$
}

(recebido: 30 de março de 2010; aceito: 29 de novembro de 2012)

\begin{abstract}
RESUMO: A caracterização anatômica, física, mecânica e química da madeira fornece informações importantes para sua melhor utilização. Contudo, para que madeiras se tornem boa opção para o mercado de pisos, adicionalmente é necessária a realização de ensaios que simulem suas reais condições em serviço. Esses ensaios simulam o pisoteio executado pelos sapatos de salto com pequenas áreas de pressão, o arraste e a queda de objetos, a resistência à abrasão da superfície e o atrito oferecido durante o deslocamento de pessoas que caminham sobre ele. Grande dificuldade da seleção de novas madeiras para pisos está na ausência de valores de referência físico-mecânicos. O presente trabalho visou a caracterizar as madeiras de Eucalyptus clöeziana $\mathrm{F}$. Muell, de Eucalyptus microcorys F. Muell e de Corymbia maculata Hook, para as propriedades de densidade básica, retratibilidade, aplicação de carga rolante, de atrito estático e dinâmico, endentação causada por cargas aplicadas em pequenas áreas, impacto da esfera de aço cadente e resistência à abrasão. Foi observado que as madeiras estudadas podem ser utilizadas para a confecção de pisos, de acordo com seus resultados obtidos e por meio de comparações com resultados de literatura.
\end{abstract}

Palavras-chave: Piso de madeira, ensaio mecânico em pisos, propriedade físico-mecânica.

\section{SIMULATION OF THE USE OF WOODEN FLOORS OF Eucalyptus sp AND Corymbia maculata}

\begin{abstract}
The anatomic, physical, mechanical and chemical characterization of wood provides important information for the indication of its best usage. However, to make these woods possible to become a good option in the wooden floors market, it is necessary to perform tests that simulate the real conditions of their practical use. These tests simulate the stomping of high-heeled shoes with small pressure areas, the dragging and falling of objects, the resistance to the surface abrasion and the friction offered during the walking of people. The difficulty of selection of new wood to manufacture floors is in the absence of specific physical-mechanical reference values. The present work intended to characterize the Eucalyptus clöeziana F. Muell, Eucalyptus microcorys F. Muell and Corymbia maculata Hook woods, regarding the properties of basic density, shrinkage, application of rolling roll, of static and dynamic friction, denting caused by loads applied on small areas, impact offalling steel sphere and resistance to abrasion. It was noticed that the woods may be used to the manufacturing of floors, according to results obtained and through comparisons with literature results.
\end{abstract}

Key words: Wooden floor, mechanical test on floors, physical-mechanical properties.

\section{INTRODUÇÃO}

Os pisos de madeira na forma de laminados e sólidos demonstraram evolução no crescimento, tanto na produção como no consumo. O crescimento, no período de 1999 a 2008, foi de $140 \%$ e $186,8 \%$, respectivamente. Para o crescimento anual, os valores atingiram $10,2 \%$ e $12,4 \%$ ao ano (ASSOCIAÇÃO BRASILEIRA DA INDÚSTRIA DA MADEIRA PROCESSADA MECANICAMENTE - ABIMCI, 2008).
Apesar da grande diversidade de madeiras tropicais brasileiras disponíveis no mercado, poucas espécies são destinadas para pisos. Entre as espécies comumente utilizadas podem ser citadas o cumaru, jatobá, peroba-rosa e ipê. Essas madeiras apresentam qualidade para a finalidade em questão, mas sua utilização apresenta como desvantagem a degradação do ambiente, uma vez que a grande maioria da extração de madeira nativa é predatória.

\footnotetext{
${ }^{1}$ Engenheiro Florestal, Mestre em Ciência e Tecnologia da Madeira - Universidade Federal de Lavras/UFLA - Departamento de Ciências Florestais - Cx. P. 3037 - 37200-000 - Lavras, MG, Brasil - marcelomartins31@ yahoo.com.br

${ }^{2}$ Engenheiro Florestal, Professor Dr. em Engenharia Florestal - Universidade Federal de Lavras/UFLA - Departamento de Ciências Florestais - Cx. P. 3037 - 37200-000 - Lavras, MG, Brasil - jreinaldo@def.ufla.br

${ }^{3}$ Engenheiro Florestal, Professor Ph.D. em Ciência e Tecnologia da Madeira - Universidade Federal de Lavras/UFLA - Departamento de Ciências Florestais - Cx. P. 3037 - 37200-000 - Lavras, MG, Brasil - jtlima@ dcf.ufla.br

${ }^{4}$ Engenheiro Mecânico, Professor Doutor em Engenharia Mecânica - Universidade Estadual Paulista "Júlio de Mesquita Filho" Campus Experimental de Itapeva - Rua Geraldo Alckmin, n 519, Nossa Senhora de Fátima - 18409-010 - Itapeva, SP, Brasil tadeu@itapeva.unesp.br

${ }^{5}$ Engenheiro Florestal, Professor Mestre em Ciência e Tecnologia da Madeira - Instituto Federal de Educação Ciência e Tecnologia do Norte de Minas Gerais - Escola Agrotécnica Federal de Januária - 39480-000 - Januária, MG, Brasil - petusk@pop.com.br
} 
Para minimizar a extração de espécies nativas, destinadas à produção de madeira serrada, podem-se introduzir no mercado novas espécies potenciais de rápido crescimento oriundas de reflorestamento. Os gêneros Eucalyptus e Corymbia podem ser utilizados para produção de madeira serrada e apresentam grande variabilidade de propriedades físico-mecânicas. É importante ressaltar que mesmo os plantios sustentáveis de Eucalyptus e Corymbia proporcionam impactos ambientais, mas que são, sem dúvida, inferiores aos provocados pela extração predatória de madeira.

Embora a produção brasileira dos pisos de madeira tenha apresentado crescimento constante nos últimos anos, os pisos de madeira maciça ainda são conhecidos por um padrão de qualidade inadequado e competem no mercado consumidor, usando, principalmente, a estratégia de preços. Esse perfil decorre, principalmente, da desorganização do setor e da ausência de um padrão referencial para a qualidade do produto (JANKOWSKY et al., 2004). Para suprir a carência de padronização, confirmar o potencial e, ao mesmo tempo, identificar outras espécies de Eucalyptus e Corymbia para a produção de pisos, é necessária a realização de ensaios em condições que simulem essa forma de utilização. A norma ASTM D 2394-83 (AMERICAN SOCIETY FOR TESTING AND MATERIALS - ASTM, 1994) contempla os ensaios de cargas aplicadas em pequenas áreas, de carga rolante, da esfera de aço cadente e a determinação dos coeficientes de atrito estático e dinâmico das superfícies dos pisos. Tais ensaios simulam pisoteio executado por sapatos de saltos com pequenas áreas de contato, o rolamento e a queda de objetos sobre os pisos e determinam os coeficientes de atrito oferecidos durante o deslocamento de pessoas, sobre os pisos, respectivamente.

A resistência à abrasão e a caracterização física de madeira não são contempladas pela norma ASTM D 239483 (ASTM, 1994) como fundamentais para a determinação da qualidade de pisos.

No presente trabalho, objetivou-se caracterizar as madeiras de Eucalyptus clöeziana F. Muell, Eucalyptus microcorys F. Muell e Corymbia maculata Hook quanto às suas propriedades físicas e de resistência mecânica simuladas em condições de uso em pisos, comparando com dados de literatura das madeiras normalmente utilizadas para esse fim.

\section{MATERIAL E MÉTODOS}

Foram utilizadas madeiras de Eucalyptus clöeziana F. Muell, Eucalyptus microcorys F. Muell e Corymbia maculata Hook, de diferentes procedências.

As árvores de E. microcorys e C. maculata, com diâmetros médios de 40,8 e 34,4 cm e alturas médias de 37 e $30 \mathrm{~m}$, respectivamente, eram provenientes de teste de procedências (PRODEPEF), implantado em 1975, na Universidade Federal de Lavras. Já, a madeira de $E$. clöeziana foi fornecida pela Preservar Madeira Reflorestada Ltda. As árvores possuíam diâmetros médios $\left(D_{1,3 \mathrm{~m}}\right)$ altura de $30 \mathrm{~cm}$ e $25 \mathrm{~m}$, respectivamente.

Na unidade experimental de desdobro e secagem da madeira (DCF/UFLA), as toras foram desdobradas e as tábuas, de aproximadamente $3000 \times 25 \mathrm{~mm}$ (comprimento $\mathrm{x}$ espessura) e largura variável, foram secas em uma estufa convencional (BARBOSA, 2003). Paralelamente, retiraram-se corpos-de-prova nos diâmetros mensurados a $1,30 \mathrm{~m}$ do solo denominados $\mathrm{D}_{1,3 \mathrm{~m}}$ para a determinação da densidade, contrações e coeficientes de anisotropia, segundo as normas NBR 11941 e NBR 7190, respectivamente (ASSOCIAÇÃO BRASILEIRA DE NORMAS TÉCNICAS - ABNT, 1997, 2003).

No laboratório de usinagem da madeira (DCF/ UFLA), preparou-se 1200 corpos-de-prova de 240 x 120 x $20 \mathrm{~mm}$, que foram transportados para uma câmara climática $\left[\mathrm{T}=(20 \pm 2)^{\circ} \mathrm{C}\right.$ e UR $\left.=(60 \pm 5) \%\right]$, atingindo umidade de $12 \%$, condição em que realizaram os ensaios de simulação em serviço (ASTM, 1994), como o impacto da esfera de aço cadente, a carga rolante, a endentação causada por cargas aplicadas em pequenas áreas e os atritos estático e dinâmico. Para tanto, utilizaram-se máquinas descritas por Padilha et al. (2006), com sistema de automação de deslocamentos das cargas. As medições das depressões ou endentações obtidas nos ensaios foram realizadas por meio de relógio comparador $(0,001 \mathrm{~mm}$ de precisão). O ensaio de resistência à abrasão foi desenvolvido no Taber Abraser, modelo 5135 (Figura 1). Foram confeccionados 24 corpos-de-prova de 95 x 95 x $20 \mathrm{~mm}$, por espécie. Foram utilizados abrasivos H-18, cargas de 500 g por braço, frequência de rotação de 72 ciclos* minn $^{-1}$ e 500 ciclos de abrasão.

Foram realizadas as análises de variância e, quando e se significativo, os testes de médias de ScottKnott, a 5\% de significância. No ensaio da esfera de aço cadente foi calculado o índice de endentação, segundo recomendação da norma ASTM D 2394- 83 (ASTM, 1994). 


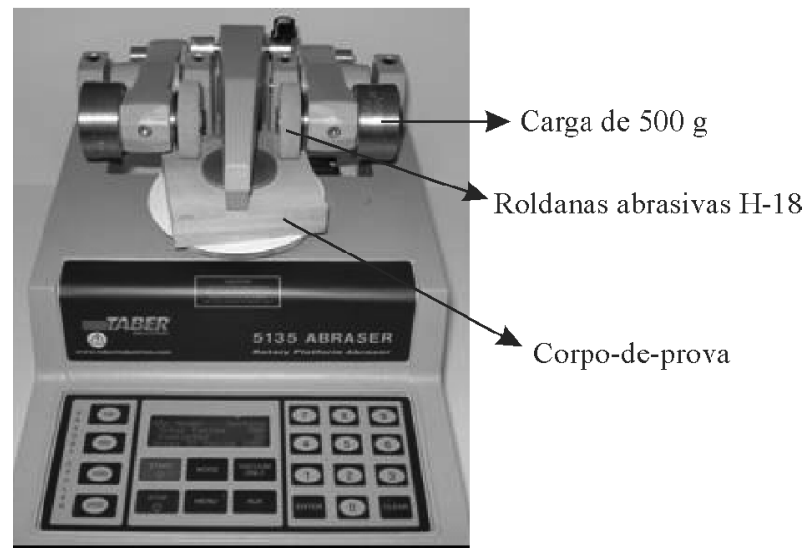

Figura 1 - Taber Abraser modelo 5135.

Figure 1 - Taber Abraser model 5135.

\section{RESULTADOS E DISCUSSÃO}

As madeiras apresentaram densidade básica superior a $0,720 \mathrm{~g}^{*} \mathrm{~cm}^{-3}$, que de acordo com a classificação do Instituto de Pesquisas Tecnológicas do Estado de São Paulo - IPT (1985) podem ser consideradas como madeiras pesadas. Observou-se a diferença estatística, a 5\% de significância, para as três espécies. A comparação múltipla entre as médias (Tabela 1) apresentou a madeira do $C$. maculata e E. microcorys com densidade básica maior que o E. clöeziana.

Tabela 1 - Comparação múltipla das médias das densidades básicas.

Table 1 - Multiple comparison of basic density averages.

\begin{tabular}{lc}
\hline Espécies de madeiras & Densidade básica $\left(\mathrm{g}^{*} \mathrm{~cm}^{-3}\right)$ \\
\hline Corymbia maculata & $0,805 \mathrm{a}$ \\
Eucalyptus microcorys & $0,795 \mathrm{a}$ \\
Eucalyptus clöeziana & $0,740 \mathrm{~b}$ \\
\hline
\end{tabular}

Médias seguidas de, pelo menos, uma mesma letra não diferem entre si a 5\% de significância, pelo teste de Scott-Knott.
Carmo (1996) obteve densidade básica média de $0,750 \mathrm{~g}^{*} \mathrm{~cm}^{-3}$ para as madeiras de E. clöeziana e $C$. maculata, com a idade de 21 anos. Tais valores de densidade básica são bem próximos aos valores encontrados para a madeira de E. clöeziana e menores que os encontrados para $C$. maculata. O valor médio da densidade básica da madeira de E. microcorys foi superior ao encontrado por Brito et al. (1983) que obteve valor médio de $0,583 \mathrm{~g}^{*} \mathrm{~cm}^{-3}$ para a mesma espécie, porém com 10 anos de idade.

Grandes variações dimensionais em madeiras, destinadas à confecção de pisos, causam sérios problemas. Esses defeitos aumentam o custo de manutenção e comprometem a estética e a segurança de pisos de madeira. Os valores médios de contrações lineares, contrações volumétricas e coeficiente de anisotropia estão listados na Tabela 2.

A madeira de E. clöeziana e de E. microcorys apresentaram valores de contrações lineares médios inferiores ao $C$. maculata. De acordo com os valores dos coeficientes de anisotropia, observa-se que a madeira de C. maculata apresentou-se menos propensa a defeitos oriundos da secagem, com melhor estabilidade dimensional, característica importante para uso da madeira como pisos. Por outro lado, a contração volumétrica da madeira de $C$. maculata foi superior às outras espécies estudadas.

Na Tabela 3, encontram-se os valores das depressões causadas pelo ensaio de impacto da esfera de aço cadente. Observa-se que não houve diferença estatística, a 5\% de significância.

Observa-se que aumentos na densidade da madeira promovem reduções nas depressões. Carmo (1996), trabalhando com Eucalyptus urophylla (21 anos), encontrou depressões médias de $0,53 \mathrm{~mm}$, que apresentouse de qualidade inferior. Para madeiras de Cumaru, Jatobá e Ipê, usualmente empregadas em pisos, a mesma autora encontrou depressões de 0,33, 0,23 e 0,32 mm,

Tabela 2 - Comparação múltipla das médias das contrações tangencial, radial, volumétrica e coeficientes de anisotropia.

Table 2 - Multiple comparisons of average values of tangential, radial and volumetric shrinkage and anisotropy coefficients.

\begin{tabular}{lcccc}
\hline \multirow{2}{*}{ Espécies de madeiras } & \multicolumn{3}{c}{ Contração $(\%)$} & \multirow{2}{*}{ Coeficiente de anisotropia } \\
\cline { 2 - 4 } & Tangencial & Radial & Volumétrica & 1,9 \\
\hline E. clöeziana & $9,8 \mathrm{a}$ & $5,2 \mathrm{a}$ & $14,8 \mathrm{a}$ & 1,5 \\
E. microcorys & $9,8 \mathrm{a}$ & $6,5 \mathrm{a}$ & $16,7 \mathrm{~b}$ & 1,2 \\
C. maculata & $10,4 \mathrm{~b}$ & $8,6 \mathrm{~b}$ & $18,3 \mathrm{c}$ & \\
\hline
\end{tabular}

Médias seguidas de, pelo menos, uma mesma letra na coluna não diferem entre si a 5\% de significância, pelo teste de Scott-Knott. 
Tabela 3 - Comparação múltipla dos valores médios das depressões do ensaio de impacto da esfera de aço cadente.

Table 3 - Multiple comparisons of average values of holes from the impact of falling steel sphere test.

\begin{tabular}{lcc}
\hline Espécies de madeiras & $\begin{array}{c}\text { Densidade } \\
\text { básica } \\
\left(\mathrm{g}^{*} \mathrm{~cm}^{-3}\right)\end{array}$ & $\begin{array}{c}\text { Médias das } \\
\text { depressões (mm) }\end{array}$ \\
\hline Corymbia maculata & 0,805 & $0,206 \mathrm{a}$ \\
Eucalyptus microcorys & 0,795 & $0,213 \mathrm{a}$ \\
Eucalyptus clöeziana & 0,740 & $0,265 \mathrm{a}$ \\
\hline
\end{tabular}

Médias seguidas de, pelo menos, uma mesma letra na vertical não diferem entre si a 5\% de significância, pelo teste de ScottKnott.

respectivamente. Padilha et al. (2006) encontraram valores médios de depressões de 0,352 a $0,585 \mathrm{~mm}$, em diferentes regiões amostradas, para clones E. urophylla. Os autores justificaram os valores altos de depressão pela idade do material que era de 8 anos.

Na Tabela 4, encontram-se os valores médios de depressões da carga rolante, após a $10^{\mathrm{a}}, 25^{\mathrm{a}} \mathrm{e} 50^{\mathrm{a}}$ viagens.

Tabela 4 - Comparação múltipla das médias para as valores médios das depressões obtidas pelo ensaio de carga rolante, para 10,25 e 50 viagens.

Table 4-Multiple comparison of average values of holes from the rolling roll test for 10,25 and 50 travellings.

\begin{tabular}{lccc}
\hline \multirow{2}{*}{$\begin{array}{l}\text { Espécies } \\
\text { de madeiras }\end{array}$} & \multicolumn{4}{c}{ Médias das depressões $(\mathrm{mm})$} \\
\cline { 2 - 5 } & 10 viagens & 25 viagens & 50 viagens \\
\hline $\begin{array}{l}\text { Eucalyptus } \\
\text { microcorys }\end{array}$ & $0,080 \mathrm{a}$ & $0.112 \mathrm{a}$ & $0,154 \mathrm{a}$ \\
$\begin{array}{l}\text { Corymbia } \\
\text { maculata }\end{array}$ & $0,085 \mathrm{a}$ & $0,112 \mathrm{a}$ & $0,128 \mathrm{a}$ \\
$\begin{array}{l}\text { Eucalyptus } \\
\text { clöeziana }\end{array}$ & $0,128 \mathrm{~b}$ & $0,157 \mathrm{~b}$ & $0,190 \mathrm{~b}$ \\
\hline
\end{tabular}

Médias seguidas de, pelo menos, uma mesma letra na vertical não diferem entre si a 5\% de significância, pelo teste de ScottKnott.

Após as $10^{\mathrm{a}}, 25^{\mathrm{a}}$ e $50^{\mathrm{a}}$ viagens, somente a depressão ocorrida na madeira de E. clöeziana foi considerada estatisticamente diferente. Comparativamente, os dados de Padilha et al. (2006), as médias de depressões encontradas após a $50^{\mathrm{a}}$ viagem, mostraram madeira de melhor qualidade, pois esses autores apresentaram valores médios de 3,00 mm para madeiras de clones E. urophylla aos 8 anos de idade. Essa diferença é decorrente da menor densidade do material utilizado pelos autores. Carmo (1996) obteve valores médios próximos de 2,24 mm de depressão para a madeira de E. urophylla após a $50^{\mathrm{a}}$ viagem. É importante salientar que quanto maior a depressão menor a resistência mecânica da madeira.

Segundo Shedley (2002), pisos de painéis com três lâminas de Eucalyptus globulus produziram depressões de $0,23 \mathrm{~mm}$, após $25^{\mathrm{a}}$ viagem e de $0,27 \mathrm{~mm}$ após $50^{\mathrm{a}}$ viagem. Esses valores apresentam a mesma magnitude dos encontrados no presente trabalho.

Na Tabela 5, encontra-se a comparação múltipla entre as médias dos coeficientes de atrito estático e dinâmico, a 5\% de significância pelo teste de Scott-Knott.

Tabela 5 - Comparação múltipla das médias dos coeficientes de atrito estático e dinâmico das espécies estudadas.

Table 5 - Multiple comparison of average values of static and dynamic friction coefficients of the studied species.

\begin{tabular}{lcc}
\hline \multirow{2}{*}{ Espécies } & \multicolumn{2}{c}{ Coeficiente de atrito } \\
\cline { 2 - 3 } & Estático & Dinâmico \\
\hline Eucalyptus clöeziana & $0,25 \mathrm{a}$ & $0,15 \mathrm{a}$ \\
Eucalyptus microcorys & $0,28 \quad \mathrm{~b}$ & $0,13 \mathrm{a}$ \\
Corymbia maculata & $0,34 \quad \mathrm{c}$ & $0,19 \mathrm{~b}$ \\
\hline
\end{tabular}

Médias seguidas de, pelo menos, uma mesma letra na coluna não diferem entre si a 5\% de significância, pelo teste de Scott-Knott.

Observa-se que a média dos coeficientes de atrito estático da madeira foram considerados estatisticamente diferentes entre si. Já, para as médias dos coeficientes de atrito dinâmico E. microcorys e E. clöeziana foram consideradas estatisticamente iguais e diferentes da madeira de C. maculata. Padilha et al. (2006), pesquisando sete clones de $E$. urophylla encontraram valores superiores de atrito estático $(0,38)$ e dinâmico $(0,23)$. Considerando que ainda não existe padronização de valores mínimos e máximos permitidos e que são apenas usados como referência para evitar queda de pessoas sobre os pisos causadas por escorregamentos, possibilitando, assim, trânsito seguro, pode-se denotar pequena diferença relativa. Contudo, todas as madeiras estudadas apresentaram seus coeficientes de atrito aceitáveis.

O ensaio de endentação causado por cargas aplicadas em pequenas áreas simula a compressão sobre pisos por saltos de sapatos de pessoas caminhando, principalmente aquelas do sexo feminino que utilizam saltos altos com pequenas áreas de contato sobre o piso. 
Na Tabela 6, encontra-se a comparação múltipla entre as médias, a 5\% de significância, pelo teste de Scott-Knott.

Tabela 6 - Comparação múltipla das médias para os valores médios das endentações causadas por cargas aplicadas em pequenas áreas.

Table 6 - Multiple comparisons of average values of denting caused by loads applied on small areas.

\begin{tabular}{lc}
\hline Espécies de madeiras & $\begin{array}{c}\text { Médias das endentações } \\
(\mathrm{mm})\end{array}$ \\
\hline Eucalyptus clöeziana & $0,023 \mathrm{a}$ \\
Corymbia maculata & $0,043 \mathrm{~b}$ \\
Eucalyptus microcorys & $0,050 \mathrm{c}$
\end{tabular}

Médias seguidas de, pelo menos, uma mesma letra não diferem entre si a 5\% de significância, pelo teste de Scott-Knott.

Observa-se que a madeira do E. clöeziana apresentou, em média, o melhor comportamento, pois obteve a menor endentação após a aplicação de cargas em pequenas áreas. Já, a madeira de E. microcorys apresentou estatisticamente comportamento inferior à madeira do $C$. maculata. Padilha et al. (2006) encontram valores de endentação para madeiras de E. urophylla, com oito anos de idade, entre 0,039 e 0,092 mm. Observa-se que essas madeiras apresentaram endentação média superiores, indicando comportamentos piores.

A resistência à abrasão é importante para pisos em geral. Maiores resistências à abrasão de pisos de madeira proporcionam menor desgaste, que aumenta sua vida útil após o pisoteio. Os valores de resistência a abrasão estão apresentados na Tabela 7.

Tabela 7 - Profundidades médias obtidas pelo ensaio de resistência a abrasão.

Table 7 - Mean depth values obtained from the abrasion resistance test.

\begin{tabular}{lc}
\hline Espécies & Profundidade $(\mathrm{mm})$ \\
\hline Eucalyptus clöeziana & $0,055 \mathrm{a}$ \\
Eucalyptus microcorys & $0,073 \mathrm{a}$ \\
Corymbia maculata & $0,076 \mathrm{a}$ \\
\hline
\end{tabular}

Médias seguidas de, pelo menos, uma mesma letra não diferem entre si a 5\% de significância, pelo teste de Scott-Knott.

Os valores médios das depressões obtidas foram de 0,055 $\mathrm{mm}$ para o E. Clöeziana, 0,073 $\mathrm{mm}$ para o $E$. microcorys e $0,076 \mathrm{~mm}$ para o $C$. maculata. Observa-se que não houve diferença significativa para as madeiras analisadas, a 5\% de significância.

\section{CONCLUSÕES}

A densidade básica da madeira de E. clöeziana $\mathrm{F}$. Muell foi inferior às madeiras de $C$. maculata Hook e $E$. microcorys F. Muell.

As madeiras de E. clöeziana F. Muell e $E$. microcorys $\mathrm{F}$. Muell apresentaram menores percentuais de contração.

As madeiras de E. clöeziana F. Muell, E. microcorys F. Muell e C. maculata Hook, comparativamente à literatura, podem ser utilizadas para pisos.

\section{REFERÊNCIAS}

AMERICAN SOCIETY FOR TESTING AND

MATERIALS. D 2394-83. Philadelphia, 1994. 608 p.

\section{ASSOCIAÇÃO BRASILEIRA DA INDÚSTRIA DA}

MADEIRA PROCESSADA MECANICAMENTE. Estudo

setorial 2007. Curitiba, 2008. 52 p.

ASSOCIAÇÃO BRASILEIRA DE NORMAS TÉCNICAS.

NBR 7190: projetos de estruturas de madeira. Rio de Janeiro, 1997. 107 p.

\section{ASSOCIAÇÃO BRASILEIRA DE NORMAS TÉCNICAS.}

NBR 11941: madeira: determinação da densidade básica. Rio de Janeiro, 2003. 6 p.

BARBOSA, C. G. Incidência dos defeitos de secagem como índice de qualidade e de seleção genética para madeira de Eucalyptus. 2003. 71 p. Dissertação (Mestrado Ciência e Tecnologia da Madeira) - Universidade Federal de Lavra, Lavras, 2003.

BRITO, J. O.; BARRICHELO, L. E. G.; SEIXAS, F. Análise da produção energética e de carvão vegetal de espécies de eucalipto. Instituto de Pesquisas e Estudos Florestais, Piracicaba, n. 23, p. 53-56, abr. 1983.

CARMO, A. P. T. Avaliação de algumas propriedades da madeira de seis espécies e eucalipto. 1996. 74 f. Dissertação (Mestrado em Ciência e Tecnologia da Madeira) - Universidade Federal de Viçosa, Viçosa, MG, 1996. 
INSTITUTO DE PESQUISAS TECNOLÓGICAS DO ESTADO DE SÃO PAULO. Ensaios físicos e mecânicos: madeiras ensaiadas: agrupamento de propriedades físicas e mecânicas. São Paulo, 1985.

JANKOWSKY, I. P.; LUIZ, M. G.; ANDRADE, A. Pisos de madeira maciça: agregando valor e qualidade ao produto. In: ENCONTRO BRASILEIRO EM MADEIRAS E EM ESTRUTURAS DE MADEIRA, 9., 2004, Cuiabá. Anais... Cuiabá: UFMG, 2004. 1 CD-ROM.
PADILHA, C.; LIMA, J. T.; SILVA, J. R. M.; TRUGILHO, P. F.; ANDRADE, H. B. Avaliação da qualidade da madeira de Eucalyptus urophylla para utilização em pisos. Instituto de Pesquisas e Estudos Florestais, Piracicaba, n. 71, p. 141147, 2006.

SHEDLEY, P. The cost benefits of small log processing: laminated three-ply flooring: a case study in WA. Carine, 2002. 127 p. (RIRDC Publication, 2/120. Project PN99). 